\title{
Proton-Transfer Tautomerism in 10-Hydroxybenzo[ $h]$ quinolines: Heavy Atom Effects and Non-Hydrogen-Bonded Photorotamer Formation in 77 K Glassy Matrixes
}

\author{
Pi-Tai Chou, ${ }^{*} \dagger$ Guo-Ray Wu, ${ }^{\ddagger}$ Yun-I Liu, ${ }^{\ddagger}$ Wei-Shan Yu, ${ }^{\ddagger}$ and Chau-Shuen Chiou \\ Department of Chemistry, National Taiwan University, Taipei, Taiwan ROC, and Department of Chemistry, \\ The National Chung-Cheng University, Chia Yi, Taiwan ROC
}

Received: January 30, 2002; In Final Form: April 18, 2002

\begin{abstract}
The low-lying triplet states of 10-hydroxybenzo[h] quinoline (HBQ) and its halogenated derivatives 7,9-diiodo10-hydroxybenzo[h]- quinoline (DIHBQ) in an excited-state intramolecular proton-transfer (ESIPT) process have been investigated. For DIHBQ, which is enhanced by the intramolecular heavy atom effect, the protontransfer tautomer (i.e., the keto form) phosphorescence that is maximized at $735 \mathrm{~nm}\left(\tau_{\mathrm{p}}=1.75 \mu \mathrm{s}\right)$ was resolved in a $77 \mathrm{~K}$ methylcyclohexane $(\mathrm{MCH})$ glass. Further transient absorption and ${ }^{1} \mathrm{O}_{2}$ sensitization experiments allow us to deduce the population yield and radiative decay rate of the keto triplet state to be 0.85 and $8.8 \mathrm{~s}^{-1}$, respectively. Upon increasing the excitation intensity, photolysis reactions were observed for both HBQ and DIHBQ in the MCH glass. The reversibility of the photolysis reaction throughout a thawing and freezing cycle led us to conclude that the rupture of an intramolecular hydrogen bond through the excessive energy dissipated does occur. The product exhibits a non-hydrogen-bonding type of enol emission that would otherwise be inaccessible in the hydrogen-bonded enol form because of the ultrafast ESIPT. Accordingly, relative energy levels in different spin manifolds are established during a proton-transfer cycle. The keto $\rightarrow$ enol reverse proton transfer in the lowest triplet manifold was estimated to be endergonic by $\sim 7.42 \mathrm{kcal} / \mathrm{mol}$.
\end{abstract}

\section{Introduction}

Insights into the dynamic features of a proton-transfer process have relied extensively on studies of the excited-state intramolecular proton-transfer (ESIPT) reaction. ${ }^{1-11}$ The ESIPT process generally involves transfer of a hydroxyl (or amino) proton to an acceptor such as nitrogen or a carbonyl oxygen from a preexisting hydrogen-bonding configuration, forming a proton-transfer tautomer. This unusual photophysical property (i.e., a large amount of Stokes-shifted keto $\mathrm{S}_{1}{ }^{\prime} \rightarrow \mathrm{S}_{0}{ }^{\prime}$ fluorescence (hereafter, the prime sign denotes the proton-transfer tautomer)), with a possible change of the dipole moment in either magnitude or direction during the reaction, has been found to have many important applications. Prototypical examples include dye lasers, energy/data storage devices, optical switches, ${ }^{12}$ biological probes, ${ }^{13,14}$ metal-cation chelates, ${ }^{15}$ and proton-transfer materials with future perspectives in electroluminescence. ${ }^{16}$

Among ESIPT molecules, 10-hydroxybenzo[ $h$ ]quinoline (HBQ) (see Figure 1) has very unique spectral properties. The fused 7,8-benzoquinoline structure constrains the $\mathrm{O}-\mathrm{H}-$ - - N geometry of the dominant enol form to a nearly perfect six-membered ring hydrogen-bonding configuration. Thus, HBQ possesses a very strong hydrogen bond in the ground state, as indicated by a 14.9-ppm downfield-shifted hydroxyl proton (in $\mathrm{CDCl}_{3}$ ) in the ${ }^{1} \mathrm{H}$ NMR study. ${ }^{17}$ For many ESIPT systems with relatively weak intramolecular hydrogen bonds, perturbation from polar, protic environments may modify the ESIPT dynamics, which

* Corresponding author. E-mail: chop@ccms.ntu.edu.tw

$\dagger$ National Taiwan University.

$\doteqdot$ The National Chung-Cheng University.
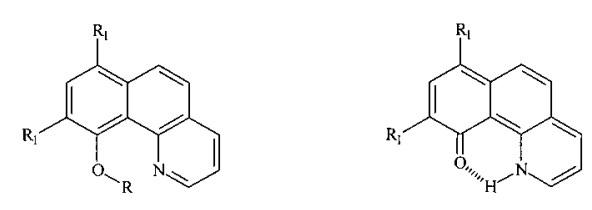

$$
\begin{array}{ll}
\text { HBQ(enol) } & \text { R: } \mathrm{H}, \quad \mathrm{R}_{1}: \mathrm{H} \\
\text { MBQ } & \text { R: } \mathrm{CH}_{3}, \mathrm{R}_{1}: \mathrm{H} \\
\text { DIHBQ } & \text { R: } \mathrm{H}, \quad \mathrm{R}_{1}: \mathrm{I} \\
\text { DIMBQ(enol)R: } \mathrm{CH}_{3}, \mathrm{R}_{1}: \mathrm{I}
\end{array}
$$

a

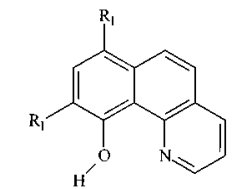

Proposed non-hydrogen-bonded enol forms of HBQ and DIHBQ

C
Figure 1. Structures of HBQ, DIHBQ, and their corresponding methoxyl derivatives.

may either be prohibited within the excited-state life span or proceed with a prerequisite of (protic) solvent reorganization. ${ }^{18}$ In contrast, the occurrence of fast ESIPT in HBQ is essentially free from solvent perturbation. For example, studies in aqueous solution have shown the occurrence of ultrafast ESIPT in HBQ throughout a wide $\mathrm{pH}$ range of $0-14 .{ }^{19}$ This data, in combination with HBQ's extraordinarily high photostability, ${ }^{17}$ leads to 
a variety of applications. In one approach, HBQ has been suggested to be a suitable radiation-hard scintillate because of its enormously large Stokes-shifted emission and high photostability. ${ }^{20}$ Sytnik et al. used HBQ to probe enzyme kinetics and concluded that HBQ could distinguish static solvent-cage polarity from dynamical solvent dielectric relaxation. ${ }^{13,14}$ Robert et al. used HBQ as a fluorescence probe to examine the influence of organized media such as cyclodextrins in aqueous solution. ${ }^{21}$ Studies on comprehensive solvatochromism effects for HBQ in a variety of solvents have been performed by del Valle and Catalán ${ }^{22}$ in an attempt to shed light on the use of HBQ in probing the solvent dynamics as well as in elucidating the biological environment. Recently, the femtosecond time-resolved approach has shown that the rate of ESIPT for enol HBQ is $>150 \mathrm{fs}^{-1}$. The results, in combination with the lack of a deuterium isotope effect on the ESIPT dynamics, suggest a barrierless ESIPT reaction along the potential energy surface. ${ }^{23}$

Despite extensive information regarding ESIPT properties in the singlet-state manifold, to our knowledge, all attempts to resolve the spectroscopy and dynamics of triplet states for HBQ are still obscured. The peak maximum of the keto-tautomer fluorescence, depending on the solvent polarity, was reported to be $>580 \mathrm{~nm} \cdot{ }^{17,19,21-23}$ One thus expects the $\mathrm{T}_{1}{ }^{\prime}-\mathrm{S}_{0}{ }^{\prime}$ energy gap for HBQ to be in the far-visible or near-infrared (NIR) regions. On one hand, correlation of the forbidden triplet $\rightarrow$ singlet radiative decay rate constant $k_{\mathrm{P}}$ generally obeys the relationship $k_{\mathrm{P}} \propto|D|^{2} \bar{v}^{3}$, where $D$ is the transition moment length and $\bar{v}$ is the average wavenumber of the transition. ${ }^{24} k_{\mathrm{P}}$ is then proportional to $E_{\mathrm{T}}^{3}$, where $E_{\mathrm{T}}$ denotes the energy gap between the $\mathrm{T}_{1}{ }^{\prime}$ and $\mathrm{S}_{0}{ }^{\prime}$ states and becomes smaller when $E_{\mathrm{T}}$ decreases to the NIR region. On the other hand, a theory pertaining to the radiationless decay derived by Siebrand concludes that the intramolecular radiationless deactivation should increase with the decreasingenergy gap of the transition. ${ }^{25}$ For many ESIPT molecules in particular, vibrational modes associated with intramolecular hydrogen bonds usually act as good quenchers for the tautomer emission. ${ }^{26}$ Thus, detecting phosphorescence in the NIR region, especially for the ESIPT molecules, may be hampered by the dominant $\mathrm{T}_{1}{ }^{\prime} \rightarrow \mathrm{S}_{1}{ }^{\prime}$ radiationless deactivation process.

In this study, the spectroscopy and dynamics of triplet states for HBQ and its halogenated derivatives were investigated. On the basis of intramolecular heavy atom effects, ${ }^{27}$ in combination with an ultrasensitive detecting system, keto-tautomer phosphoresce in the NIR region was resolved in 7,9-diiodo-10hydroxybenzo[ $h]$ quinoline (DIHBQ, Figure 1$)$. Intriguing photolysis reactions were also observed for both HBQ and DIHBQ in a $77 \mathrm{~K} \mathrm{MCH}$ glass, giving rise to the enol-like emission that would otherwise be unobtainable. As a result, relative energy levels in different spin manifolds during a proton-transfer cycle were thus established.

\section{Experimental Section}

2.1. Materials. $10-\mathrm{Hydroxybenzo}[h]$ quinoline (HBQ) (TCI Inc) was twice recrystallized from cyclohexane followed by purification using column chromatography (2:1 ethyl acetate/ hexanes). The purity was checked by using the fluorescence excitation spectrum. Methylcyclohexane (Aldrich) was of spectrograde quality and was used without further purification. No fluorescence or phosphorescence impurities were detected in the blank solvent at either room temperature or $77 \mathrm{~K}$.

DIHBQ was synthesized by adding a solution containing HBQ $(0.01 \mathrm{~mol})$ and glacial acetic acid $(4 \mathrm{~mL})$ in chloroform
$(10 \mathrm{~mL})$ to a solution of iodine $(0.02 \mathrm{~mol}$ in $20 \mathrm{~mL}$ methanol $)$ for a period of $1 \mathrm{~h}$. The precipitate was collected and neutralized with sodium carbonate to obtain DIHBQ. ${ }^{1} \mathrm{H} \mathrm{NMR}\left(\mathrm{CDCl}_{3}, 400\right.$ $\mathrm{MHz}): \delta 7.659(\mathrm{t}, J=12.72 \mathrm{~Hz}, 1 \mathrm{H}), 7.762(\mathrm{~d}, J=9.32 \mathrm{~Hz}$, $1 \mathrm{H}), 8.084(\mathrm{~d}, J=8.8 \mathrm{~Hz}, 1 \mathrm{H}), 8.338(\mathrm{~d}, J=8.4 \mathrm{~Hz}, 1 \mathrm{H})$, $8.572(\mathrm{~s}, 1 \mathrm{H}), 8.853$ (d, $J=4.6 \mathrm{~Hz}, 1 \mathrm{H}), 16.705$ (s, 1H). A general methylation procedure incorporating methyliodide into the basic DIHBQ solution to obtain 7,9-diiodo-10-methoxybenzo[ $h]$ quinoline (DIMBQ) failed because of the detachment of iodine from DIHBQ at the reflux temperature. Alternatively, 10-methoxybenzo[h]quinoline (MBQ) was first synthesized through the methylation of HBQ $(0.5 \mathrm{~g}) .{ }^{19}$ DIMBQ was then obtained by a similar iodination procedure. Crude DIMBQ was further purified by column chromatography (eluent: ethyl acetate). ${ }^{1} \mathrm{H}$ NMR $\left(\mathrm{CDCl}_{3}, 400 \mathrm{MHz}\right): \delta 4.512$ (s, 3H), 7.489 $(\mathrm{d}, J=8.0 \mathrm{~Hz}, 1 \mathrm{H}), 7.767(\mathrm{~d}, J=8.5 \mathrm{~Hz}, 1 \mathrm{H}), 7.952(\mathrm{t}, 1 \mathrm{H})$, 8.097 (s, 1H), 8.868 (d, $J=7.6 \mathrm{~Hz}, 1 \mathrm{H}), 9.630$ (d, $J=5.2 \mathrm{~Hz}$, $1 \mathrm{H})$.

2.2. Method. Steady-state absorption and fluorescence spectra were recorded by a Varian (Cary 3E) spectrophotometer and a Hitachi (F4500) fluorimeter, respectively. Detailed fluorescence lifetime measurements have been described in the previous report. $^{23}$ Briefly, the second harmonic of the $\mathrm{Ti}$-sapphire oscillator (100 fs, $82 \mathrm{MHz}$, Spectra Physics) coupled with a pulse picker (NEOS, model N17389) was used as an excitation source, giving a tunable wavelength in the range of 380-410 $\mathrm{nm}$ with a repetition rate of $8 \mathrm{MHz}$. An Edinburgh OB 900-L single photon counter was the detector, giving a temporal resolution of $\sim 15$ ps. For time-resolved phosphorescence measurements, an Nd:YAG (355 nm, 8 ns, Continuum Surlite II) pumped optical parametric oscillator was tuned between 620 and $800 \mathrm{~nm}$ and was then frequency-doubled by BBO crystals to obtain a 310-400-nm excitation frequency. The resulting luminescence was then detected by a red-sensitive intensified charge coupled detector (ICCD, Princeton Instrument, model $576 \mathrm{G} / 1$ ) coupled with a polychromator in which the grating is blazed with a maximum at $700 \mathrm{~nm}$.

The transient absorption experiment was performed by using a modified flash lamp (EG\&G model LS-1130) as a white-light probe pulse. A cylindrical lens was used to shape the pumped $\mathrm{Nd}$ :YAG 355-nm pulse to a rectangular size of $10 \times 2 \mathrm{~mm}^{2}$. The white-light probe pulse was collected by an optical fiber, collimated by a $5-\mathrm{cm}$ focal-length lens, and skimmed to a $2 \times$ $2-\mathrm{mm}^{2}$ rectangle before entering the sample cell. The pump and probe pulses were crossed at a $90^{\circ}$ angle, with an overlapping distance of $10 \mathrm{~mm}$. The probe pulse, after passing through the sample solution, was focused on the entrance slit $(\sim 700 \mu \mathrm{m})$ of the ICCD system.

Steady-state $\mathrm{O}_{2}\left({ }^{1} \Delta_{\mathrm{g}} \rightarrow{ }^{3} \Sigma_{\mathrm{g}}{ }^{-}(0,0)\right)$ emission spectra in the region of $1274 \mathrm{~nm}$ were obtained by exciting the sample solution under a front-face excitation configuration using an Ar ion laser (362 nm, Coherent Innova 90). The emission was then sent through an NIR Fourier transform interferometer (Bruker Equinox 55) and detected by a liquid nitrogen-cooled Ge detector (403X, Applied Detector Corporation). For the timeresolved study, the third harmonic $(355 \mathrm{~nm})$ of the Nd:YAG laser (Continuum Surlite I) was used as an excitation light source. An NIR fast-response photomultiplier (Hamamatsu model R5509-72) operated at $-80{ }^{\circ} \mathrm{C}$ was used as a detecting system. The response time of the overall detecting system was determined to be $\sim 20 \mathrm{~ns}$, as measured by the response of the system to a 1064-nm Nd:YAG laser pulse of 8-ns duration. To select the emission of interest, a combination of interference and band-pass filters was used. 


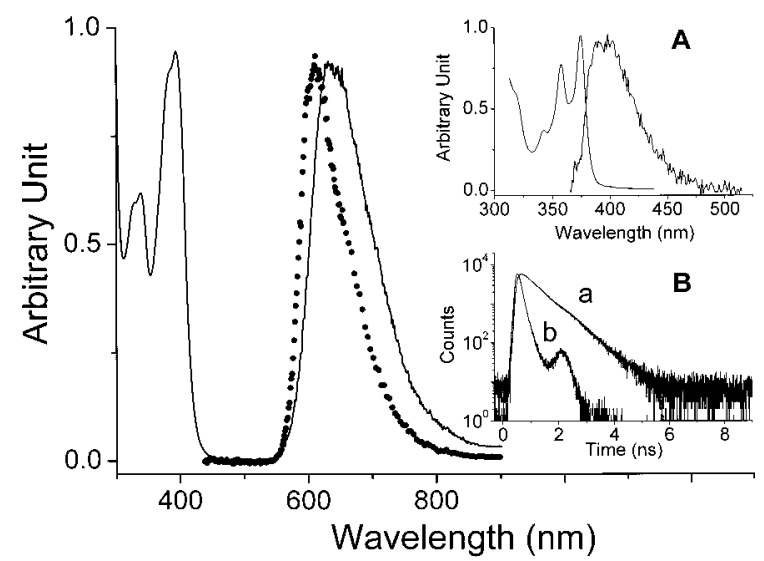

Figure 2. Absorption and emission spectra of DIHBQ (-) in $\mathrm{MCH}$

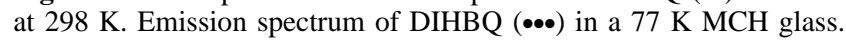
(A) Absorption and emission spectra of DIMBQ in $\mathrm{MCH}$ at $298 \mathrm{~K}$. (B) Fluorescence decay at $610 \mathrm{~nm}$ for DIHBQ at $77 \mathrm{~K}$ (a) and the system response function (b) $\left(\lambda_{\mathrm{ex}}=395 \mathrm{~nm}\right)$.

In the oxygen-dependent study, the molar ratio of oxygen in solution was determined by Henry's law from known $\mathrm{O}_{2}$ concentrations for various solvents at 760 Torr. ${ }^{28}$

\section{Results and Discussion}

3.1. Photophysics of HBQ and 7,9-DIHBQ. Figure 2 shows the room-temperature absorption and emission spectra of DIHBQ in MCH. The $\mathrm{S}_{0} \rightarrow \mathrm{S}_{1}\left(\pi \pi^{*}\right)$ absorption exhibits an onset at $\sim 440 \mathrm{~nm}$ with a peak maximum at $395 \mathrm{~nm}\left(\epsilon_{395} \approx 8100\right.$ $\mathrm{M}^{-1} \mathrm{~cm}^{-1}$ ). The luminescence is mainly characterized by a largely Stokes-shifted emission band maximized at $640 \mathrm{~nm}$. The excitation spectrum is independent of the monitored emission wavelength and is effectively identical to the absorption profile (not shown here). Conversely, DIMBQ, which is considered to be a non-proton-transfer model with an electronic configuration similar to that of DIHBQ, exhibits a very weak, normal Stokesshifted fluorescence maximum at $\sim 400 \mathrm{~nm}$ (see inset $\mathrm{A}$ of Figure 2). Thus, similar to what was concluded in HBQ, ${ }^{17,19,23}$ ESIPT is operative in DIHBQ, resulting in keto-tautomer emission (see Scheme 1).

Not detecting any enol emission for DIHBQ is consistent with the occurrence of rapid proton-transfer tautomerism in the lowest singlet excited state. This viewpoint is further supported by the fluorescence lifetime measurement where the keto-tautomer emission is well-fitted by a single-exponential decay rate of 220 $\mathrm{ps}^{-1}$, whereas the rise time is beyond the system response of 15 ps. On the basis of approximately unity ESIPT efficiency for DIHBQ, the quantum yield of the keto-tautomer emission was determined to be $1.8 \times 10^{-3}$ by comparing its emission intensity to that of HBQ $\left(\tau_{\mathrm{f}} \approx 300 \mathrm{ps}, \Phi_{\mathrm{obs}} \approx 2.1 \times 10^{-3}\right.$ in cyclohexane ${ }^{23}$ ). In comparison to the spectra of HBQ, both absorption and fluorescence spectra of DIHBQ exhibit significant red shifts of $\sim 1000$ and $400 \mathrm{~cm}^{-1}$, respectively, in cyclohexane. The bathochromic shift could be qualitatively rationalized by the electron-donating properties of iodine substituents through the resonance effect, reducing the $\pi \rightarrow \pi^{*}$ energy gap in both the enol and keto species.

In a $77 \mathrm{~K} \mathrm{MCH}$ glass, an ESIPT reaction with an ultrafast rate still takes place in DIHBQ, as was supported by two observations: (1) a unique keto-tautomer fluorescence peak that was maximized at $610 \mathrm{~nm}\left(\Phi_{\mathrm{f}} \approx 6.8 \times 10^{-3}\right)$ was resolved in a steady-state approach (Figure 2) and (2) the fluorescence lifetime was deduced to be $\sim 600 \mathrm{ps,} \mathrm{whereas} \mathrm{the} \mathrm{rise} \mathrm{time} \mathrm{is}$ still beyond our system response of $15 \mathrm{ps}$ (see inset B of Figure

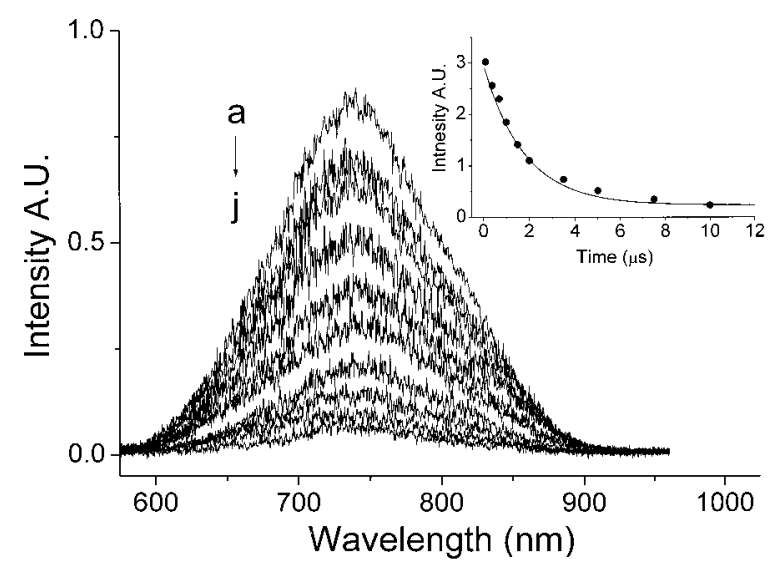

Figure 3. Time-dependent $T_{1}{ }^{\prime} \rightarrow S_{0}{ }^{\prime}$ phosphorescence spectra of DIHBQ in a $77 \mathrm{~K} \mathrm{MCH}$ glass acquired at various delay times of (a) $100 \mathrm{~ns}$, (b) $400 \mathrm{~ns}$, (c) $700 \mathrm{~ns}$, (d) $1.0 \mu \mathrm{s}$, (e) $1.5 \mu \mathrm{s}$, (f) $2.0 \mu \mathrm{s}$, (g) $3.5 \mu \mathrm{s}$, (h) 5.0 $\mu \mathrm{s}$, (i) $7.5 \mu \mathrm{s}$, and (j) $10 \mu \mathrm{s}$ with respect to the pump pulse. Inset: Decay profile of 735-nm emission intensity. Note that the spectral feature at $>800 \mathrm{~nm}$ may not be authentic because of the nonlinear spectral response of the ICCD.

\section{SCHEME 1: Relative Energy Levels (in $\mathrm{cm}^{-1}$ ) for the Enol and Keto Tautomers of DIHBQ Estimated from Either Experimental or Theoretical Approaches ${ }^{a}$}

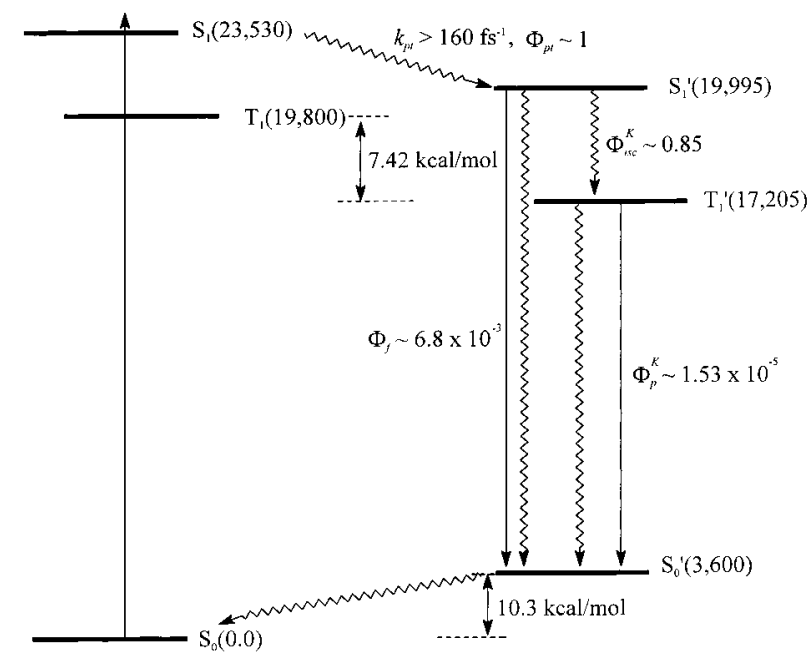

DIHBQ(enol form)

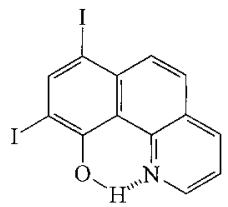

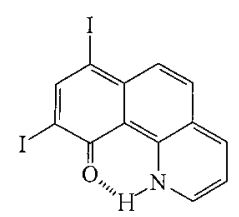

DIHBQ(keto form)
${ }^{a}$ See text for a detailed description.

2). The fluorescence peak maximum of $610 \mathrm{~nm}$ is blue-shifted by $\sim 800 \mathrm{~cm}^{-1}$ with respect to that observed at $298 \mathrm{~K}$. A similar hypsochromic shift has been reported for HBQ in a $77 \mathrm{~K} \mathrm{MCH}$ glass ${ }^{17}$ and was tentatively attributed to the solvent cage effect. To obtain the presumably ultraweak $\mathrm{T}_{1}{ }^{\prime} \rightarrow \mathrm{S}_{0}{ }^{\prime}$ phosphorescence, the high-voltage gate of the ICCD was operated at a delay time of $>50 \mathrm{~ns}$ to eliminate the fast decay component attributed to the keto-tautomer fluorescence. Upon low-power excitation $(<1$ $\mathrm{mJ} / \mathrm{cm}^{2}$, vide infra), phosphorescence at $>600 \mathrm{~nm}$ was resolved, and its spectral evolution as a function of the delay time is shown in Figure $3 \mathrm{a}-\mathrm{j}$. The long-lived emission that is maximized at $735 \mathrm{~nm}$ undergoes single-exponential decay kinetics, of which the lifetime was fitted to be $1.75 \mu \mathrm{s}\left(k_{\mathrm{obs}} \approx 5.7 \times 10^{5} \mathrm{~s}^{-1}\right.$, see 
inset of Figure 3). The excitation spectrum that is tuned in the $380-405 \mathrm{~nm}$ region revealed the same spectral profile as that monitored at the keto-tautomer fluorescence (not shown here), indicating that both emissions originate from a common ground state. In comparison, DIMBQ exhibited a phosphorescence band that was maximized at $495 \mathrm{~nm}\left(\tau_{\mathrm{p}} \approx 1.2 \mathrm{~ms}\right)$ in a $77 \mathrm{~K} \mathrm{MCH}$ glass. The results unambiguously show that the 735-nm emission originates from the keto-tautomer $\mathrm{T}_{1}{ }^{\prime} \rightarrow \mathrm{S}_{0}{ }^{\prime}$ phosphorescence. By opening the ICCD gate width as wide as $10 \mu$ s to cover $>99.9 \%$ of the phosphorescence decay, the observed yield of ketotautomer phosphorescence, $\Phi_{\mathrm{obs}}^{\mathrm{K}}$, was measured to be $1.3 \times$ $10^{-5}$. Under identical experimental conditions, our attempts to resolve the keto-tautomer phosphorescence for HBQ failed. It is thus reasonable to conclude that the phosphorescence yield of $\mathrm{HBQ}$, if it exists, should be $\ll 1.3 \times 10^{-5}$.

Theoretically, the observed yield for the keto-tautomer phosphorescence can be expressed as $\Phi_{\mathrm{obs}}^{\mathrm{K}}=\Phi_{\mathrm{pt}} \Phi_{\mathrm{isc}}^{\mathrm{K}} \Phi_{\mathrm{p}}^{\mathrm{K}}$ where $\Phi_{\mathrm{pt}}$ denotes the yield of the ESIPT and is assumed to be $\sim 100 \%$ efficient because of the ultrafast rate of ESIPT, $\Phi_{\text {isc }}^{\mathrm{K}}$ is the population yield of the keto-tautomer triplet state, and $\Phi_{\mathrm{p}}^{\mathrm{K}}$ represents the keto phosphorescence yield and is equivalent to $k_{\mathrm{r}}^{\mathrm{P}} / k_{\mathrm{obs}}^{\mathrm{P}}$ where $k_{\mathrm{r}}^{\mathrm{P}}$ and $k_{\mathrm{obs}}^{\mathrm{P}}$, respectively, denote the radiative and measured decay rate constants of the keto phosphorescence. The quantitative measurement of $\Phi_{\mathrm{isc}}^{\mathrm{K}}$ by using triplet-triplet energy transfer to an organic molecule is not feasible for DIHBQ mainly because of the lack of sensitizers with known low-lying triplet-state energies and absorption cross sections that could allow energy evaluation from quenching (i.e., energy transfer) experiments. Alternatively, we performed an oxygen photosensitization experiment to circumvent this obstacle. This method is feasible in determining the yield of the triplet state of organic molecules if the following assumptions hold: (i) The $\mathrm{T}_{1}-\mathrm{S}_{0}$ energy gap is greater than the energy required to sensitize singlet oxygen $\left(\mathrm{O}_{2}{ }^{1} \Delta_{\mathrm{g}} \rightarrow^{3} \Sigma^{-}{ }_{\mathrm{g}}(0,0)\right.$ of $\left.\sim 7850 \mathrm{~cm}^{-1}\right)$. (ii) Sensitization of oxygen by the $S_{1}$ state is negligible because of its relatively much shorter life span. (iii) The decay of the triplet state should be dominated by the $\mathrm{T}_{1}-{ }^{3} \mathrm{O}_{2}$ energy transfer. Assumptions (i) and (ii) hold for DIHBQ because of the keto $\mathrm{T}_{1}{ }^{\prime}-\mathrm{S}_{0}{ }^{\prime}$ energy gap of $\sim 13605 \mathrm{~cm}^{-1}$ and fast decay of the keto-tautomer emission at room temperature (e.g., $\sim 220$ ps in $\mathrm{MCH}$ at 298 $\mathrm{K})$.

Conventionally, the $\mathrm{O}_{2}$ quenching dynamics for the triplet state can be obtained through the triplet-triplet absorption measurements as a function of the added $\mathrm{O}_{2}$ concentration. Figure 4 depicts the transient absorption spectra of DIHBQ in degassed methylcyclohexane. The transient absorption maximum at $475 \mathrm{~nm}$ undergoes a fast single-exponential decay with a rate of $8.5 \times 10^{5} \mathrm{~s}^{-1}$ (see inset A of Figure 4). The drastic quenching dynamics of the transient absorbance upon adding $\mathrm{O}_{2}$ helped us to assign the 475-nm transient to the triplet-triplet absorption unambiguously. Inset B of Figure 4 shows a plot of decay rate as a function of $\mathrm{O}_{2}$ concentration. From the best linear leastsquares fit, we deduced an $\mathrm{O}_{2}$ quenching rate constant of $3.2 \times$ $10^{9} \mathrm{~s}^{-1}$, which within experimental error is equal to $1 / 9$ of the diffusion-controlled rate $\left(\sim 3.0 \times 10^{9} \mathrm{M}^{-1} \mathrm{~s}^{-1}\right.$ calculated from the Stokes-Einstein equation $\left.{ }^{29}\right)$ that was derived theoretically from the $\mathrm{T}-\mathrm{O}_{2}$ sensitization. Thus, under oxygen saturation $\left(1.15 \times 10^{-2} \mathrm{M}\right.$ in cyclohexane at 1 atm $\left.\mathrm{O}_{2}, 298 \mathrm{~K}{ }^{28}\right)$, the decay of the triplet state should be dominated $(>97 \%)$ by the $\mathrm{T}_{1}{ }^{\prime}-{ }^{3} \mathrm{O}_{2}$ energy transfer, thereby fulfilling requirement (iii).

Figure 5 shows the emission spectrum of the $\mathrm{O}_{2}{ }^{1} \Delta_{\mathrm{g}} \rightarrow{ }^{3} \Sigma_{\mathrm{g}}{ }^{-}$ $(0,0)$ transition at $1274 \mathrm{~nm}$ that is sensitized by DIHBQ, of which the lifetime was determined to be $\sim 21 \mu$ s in methylcyclohexane. Within experimental error, the decay dynamics

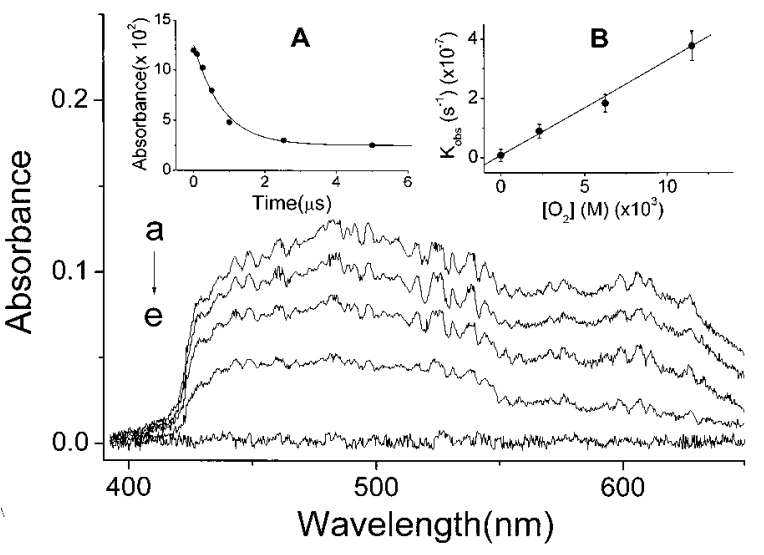

Figure 4. Transient absorption spectra of DIHBQ in degassed $\mathrm{MCH}$ (298 K) at pump - probe delay times of (a) $0 \mathrm{~ns}$, (b) $250 \mathrm{~ns}$, (c) $500 \mathrm{~ns}$, (d) $1.0 \mu \mathrm{s}$, and (e) $10 \mu \mathrm{s}$. (A) Decay kinetics of the transient absorbance monitored at $475 \mathrm{~nm}$. (B) Plot of the decay rate of transient absorbance at $475 \mathrm{~nm}$ vs oxygen concentration and the best linear least-squares fit.

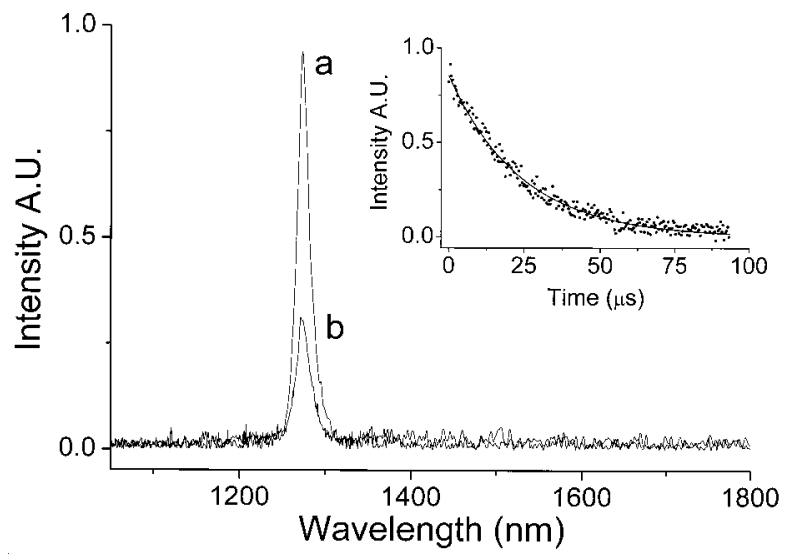

Figure 5. $\mathrm{O}_{2}{ }^{1} \Delta_{\mathrm{g}} \rightarrow{ }^{3} \Sigma_{\mathrm{g}}{ }^{-}(0,0)$ emission spectrum in $\mathrm{MCH}$ sensitized by (a) 1-H-phenalen-1-one and (b) DIHBQ under the same optical density of 0.2 at $362 \mathrm{~nm}\left(\mathrm{Ar}^{+}\right.$laser). Inset: Decay dynamics of the $\mathrm{O}_{2}$ ${ }^{1} \Delta_{\mathrm{g}} \rightarrow{ }^{3} \Sigma_{\mathrm{g}}^{-}(0,0) 1274 \mathrm{~nm}$ emission $\left(\lambda_{\mathrm{ex}}: 355 \mathrm{~nm}, \mathrm{Nd} / \mathrm{YAG}\right.$ laser $)$ sensitized by DIHBQ in MCH $(298 \mathrm{~K})$.

are identical to those of the $\mathrm{O}_{2}\left({ }^{1} \Delta_{\mathrm{g}}\right)$ emission in cyclohexane that was generated by a known sensitizer, $1-\mathrm{H}$-phenalen-1-one (PH). ${ }^{30}$ We have further determined the triplet-state population in DIHBQ by comparing its sensitized $\mathrm{O}_{2}\left({ }^{1} \Delta_{\mathrm{g}}\right)$ emission intensity with respect to that produced with the sensitizer $\mathrm{PH}$. The ratio of the sensitized $\mathrm{O}_{2}\left({ }^{1} \Delta_{\mathrm{g}}\right)$ emission intensity for DIHBQ versus $\mathrm{PH}$ was deduced to be 0.33 in $\mathrm{O}_{2}$-saturated methylcyclohexane. Counting the $\mathrm{PH}$-sensitized $\mathrm{O}_{2}\left({ }^{1} \Delta_{\mathrm{g}}\right)$ yield of $0.92 \pm 0.1^{31}$ and $\sim 98 \%$ of the triplet-state quenching dynamics for DIHBQ in $\mathrm{O}_{2}$ saturated cyclohexane (vide supra), the yield of intersystem crossing $\Phi_{\text {isc }}^{\mathrm{K}}$ was then estimated to be 0.31. In this derivation, we have assumed that the production of $\mathrm{O}_{2}\left({ }^{1} \Delta_{\mathrm{g}}\right)$ that is sensitized by the triplet state is of unit efficiency. This assumption is based on a spin statistical argument in which the only deactivation pathway of the triplet state resulting from the $\mathrm{T}_{1}{ }^{\prime}-\mathrm{O}_{2}\left({ }^{3} \Sigma_{\mathrm{g}}{ }^{-}\right)$encounter is the energy transfer to form $\mathrm{O}_{2}\left({ }^{1} \Delta_{\mathrm{g}}\right)$. Because the assumption of unit efficiency of $\mathrm{O}_{2}\left({ }^{1} \Delta_{\mathrm{g}}\right)$ generation in each $\mathrm{T}_{1}{ }^{\prime}-{ }^{3} \mathrm{O}_{2}$ encounter may be invalid for DIHBQ, we used the value of $\Phi_{\text {isc }}^{\mathrm{K}}=0.31$ as the lower limit. $\Phi_{\text {isc }}^{\mathrm{K}}$ is equivalent to $k_{\mathrm{isc}}^{\mathrm{K}} / k_{\mathrm{f}}^{\mathrm{K}}$ where $k_{\mathrm{isc}}^{\mathrm{K}}$ and $k_{\mathrm{f}}^{\mathrm{K}}$ are rate constants of intersystem crossing and fluorescence decay, respectively, for the keto form. Knowing $k_{\mathrm{f}}^{\mathrm{K}}$ to be 4.55 $\times 10^{9} \mathrm{~s}^{-1}\left(\tau_{\mathrm{f}}=220 \mathrm{ps}^{-1}\right), k_{\text {isc }}^{\mathrm{K}}$ was thus deduced to be $1.41 \times$ $10^{9} \mathrm{~s}^{-1}$ at $298 \mathrm{~K}$. $k_{\mathrm{isc}}^{\mathrm{K}}$ is further assumed to be independent of 


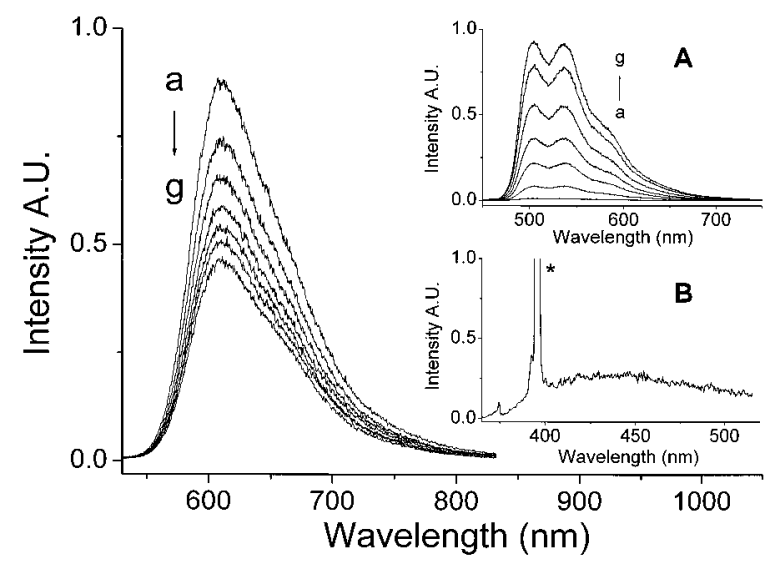

Figure 6. Disappearance of $S_{1}{ }^{\prime} \rightarrow S_{0}{ }^{\prime}$ fluorescence and growth of 505 $\mathrm{nm}$ phosphorescence spectra (A) of DIHBQ as a function of photolysis (355 nm, $35 \mathrm{~mJ} / \mathrm{cm}^{2}, 10 \mathrm{~Hz}$ ) periods of (a) 0 , (b) 0.5 , (c) 1.0 , (d) 1.5 , (e) 2.0, (f) 2.5, and (g) $3.0 \mathrm{~min}$ in a $77 \mathrm{~K} \mathrm{MCH}$ glass. (B) Fluorescence spectrum of DIHBQ after 30 min of photolysis in a $77 \mathrm{~K} \mathrm{MCH}$ glass where $>90 \%$ of DIHBQ was converted to the non-hydrogen-bonded enol form ( $\lambda_{\text {ex }}: 355 \mathrm{~nm}\left(35 \mathrm{~mJ} / \mathrm{cm}^{2}\right)$, * denotes Raman scattering).

temperature if the triplet-state population proceeds only through the $\mathrm{S}_{1}{ }^{\prime} \rightarrow \mathrm{T}_{1}{ }^{\prime}$ intersystem crossing and if the $\mathrm{S}_{1}{ }^{\prime}-\mathrm{T}_{1}{ }^{\prime}$ energy gap is $\gg k T$. Support for the latter assumption is given by the $>2700$ $\mathrm{cm}^{-1}$ gap measured from the difference in peak maxima between keto fluorescence $\left(\sim 16400 \mathrm{~cm}^{-1}\right)$ and phosphorescence $(\sim 13605$ $\left.\mathrm{cm}^{-1}\right)$. Knowing the observed decay rate of $\sim 1.67 \times 10^{9} \mathrm{~s}^{-1}$ $\left(\tau_{\mathrm{f}} \approx 600 \mathrm{ps}\right)$ for the keto fluorescence, $\Phi_{\mathrm{isc}}^{\mathrm{K}}$ and $\Phi_{\mathrm{p}}^{\mathrm{K}}$ were thus calculated to be 0.85 and $1.53 \times 10^{-5}$, respectively, for DIHBQ in a $77 \mathrm{~K} \mathrm{MCH}$ glass. According to the relationship of $\Phi_{\mathrm{p}}^{\mathrm{K}}=$ $k_{\mathrm{r}}^{\mathrm{P}} / k_{\text {obs }}^{\mathrm{P}}$ where $k_{\text {obs }}^{\mathrm{P}}$ was measured to be $5.71 \times 10^{5} \mathrm{~s}^{-1}$, the radiative decay rate of keto phosphorescence, $k_{\mathrm{r}}^{\mathrm{P}}$, was further deduced to $8.8 \mathrm{~s}^{-1}$.

3.2. Photolysis of DIHBQ and HBQ. Upon increasing the excitation laser intensity $(355 \mathrm{~nm})$ of $>30 \mathrm{~mJ} / \mathrm{cm}^{2}$, significant photolysis time-dependent spectral evolutions for DIHBQ were observed in which both fluorescence $\left(\lambda_{\max } \approx 610 \mathrm{~nm}\right)$ and phosphorescence $\left(\lambda_{\max } \approx 735 \mathrm{~nm}\right)$ of the keto tautomer gradually decreased, accompanied by the appearance of an emission band that was maximized at $505 \mathrm{~nm}$ (Figure 6). By scanning the delay time of the gating ICCD, the lifetime of the 505-nm emission was measured to be $1.52 \mathrm{~ms}$. It should be noted that after each photolysis period emission spectra and their associated lifetime of products were obtained under sufficiently low excitation intensity $\left(<1.0 \mathrm{~mJ} / \mathrm{cm}^{2}\right)$ to avoid further photolysis reactions. The long life span unambiguously allows us to assign the 505$\mathrm{nm}$ emission band to the phosphorescence. When the sample was thawed to the liquid phase and then was quickly frozen back to the glassy form at $77 \mathrm{~K}$, both the fluorescence and phosphorescence of the keto form were completely recovered, accompanied by the disappearance of the 505-nm phosphorescence. In a comparative study, DIMBQ in a $77 \mathrm{~K}$ MCH glass exhibited phosphorescence with spectral features and relaxation dynamics $\left(\lambda_{\max } \approx 495 \mathrm{~nm}, \tau_{\mathrm{p}} \approx 1.2 \mathrm{~ms}\right.$ and $\left.\Phi_{\mathrm{p}} \sim 0.3\right)$ that were similar to those of the photolysis product in DIHBQ. The results, in combination with the reversibility of the photolysis reaction during a thawing-and-freezing cycle, led us to conclude that upon the photolysis of DIHBQ the rupture of an intramolecular hydrogen bond takes place, forming a non-hydrogenbonded enol conformer (vide infra).

A similar photolysis reaction was observed for HBQ in the glass matrixes where the decrease of the 607-nm keto fluorescence was obvious (Figure 7). However, instead of a dominant 505-nm enol phosphorescence observed upon photolyzing

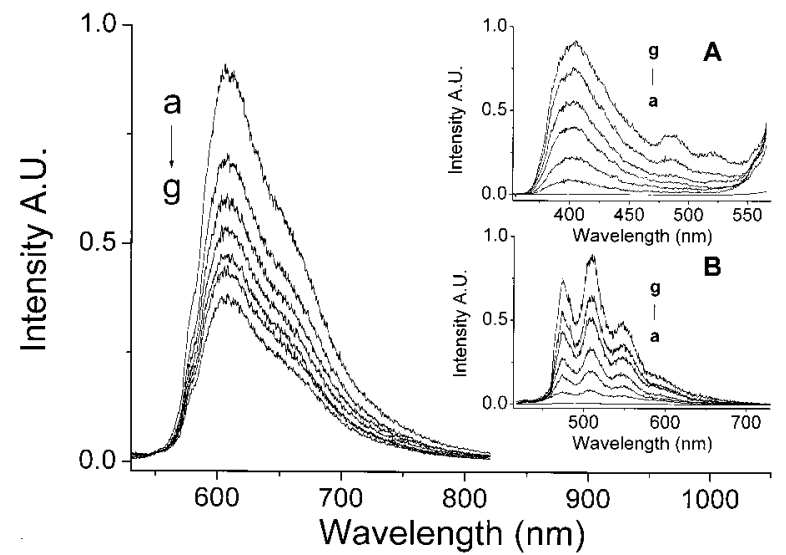

Figure 7. Disappearance of the $S_{1}{ }^{\prime} \rightarrow S_{0}{ }^{\prime}$ fluorescence and the growth of product emission (A) for HBQ in a $77 \mathrm{~K} \mathrm{MCH}$ glass under the same photolysis condition as those used in Figure 6. (B) Growth of product phosphorescence as a function of photolysis period. To acquire $\mathrm{B}$, the high-voltage gate width of ICCD was opened as wide as $10 \mathrm{~ms}$ with a delay time of $100 \mathrm{~ns}$ to eliminate fluorescence interference.

DIHBQ, the photolysis time-dependent spectral growth consists of dual emission maxima at 403 and $475 \mathrm{~nm}$ (see inset A of Figure 7). Note for inset A of Figure 7 that the ICCD was operated in a free-run mode so that the spectra were obtained via a steady-state manner. When the gate was opened at, for example, $10 \mathrm{~ms}$ followed by scanning the delay time of acquisition, long-lived 475-nm phosphorescence was resolved, the lifetime of which was determined to be $\sim 1.2 \mathrm{~s}$ (see inset $\mathrm{B}$ of Figure 7). Conversely, the lifetime of the 403-nm emission band was measured to be $\sim 3.0$ ns so that its assignment to a fluorescence band is unambiguous. We have made a further attempt to estimate the yield of the photolysis reaction. In a 77 $\mathrm{K}$ transparent MCH glassy matrix, DIHBQ (optical density $\sim 0.5$ at $355 \mathrm{~nm}$ ) was irradiated by a $355-\mathrm{nm}$ laser beam $(10 \mathrm{~Hz})$, the intensity and beam diameter of which were measured to be 2.5 $\mathrm{mJ}$ and $3.0 \mathrm{~mm}\left(\sim 35 \mathrm{~mJ} / \mathrm{cm}^{2}\right)$, respectively. After a photolysis period of $30 \mathrm{~s}$, the intensity of the keto-tautomer fluorescence was reduced by $16.4 \%$. By neglecting the inner filter effect, the photolysis efficiency $\Phi_{\text {rxn }}$ can thus be deduced from $\left(F_{0}-\right.$ $F) \%=(E \%)\left[1-\left(1-\Phi_{\text {rxn }}\right)^{n}\right]$ where $\left(F_{0}-F\right) \%$ is the percentage decrease of the keto-tautomer emission intensity and $(E \%)$ and $n$ denote the percentage of DIHBQ being excited $(\sim 45 \%)$ and the number of laser shots during the photolysis period (300), respectively. $\Phi_{\text {rxn }}$ was thus calculated to be $0.13 \%$ for DIHBQ. A similar procedure gave $\Phi_{\text {rxn }}$ to be $\sim 0.15 \%$ for HBQ.

Under the weak excitation intensity that was used to obtain the enol-like phosphorescence that is shown in inset A of Figure 6 , the fluorescence associated with the non-hydrogen-bonded enol product of DIHBQ was negligibly small. The result can be rationalized by the dominant rate of intersystem crossing that is enhanced by the iodine heavy-atom effect in the enol form. This viewpoint can be further supported by the ultraweak enol fluorescence $\left(\Phi_{\mathrm{f}} \approx 10^{-4}, \tau_{\mathrm{f}} \approx 120 \mathrm{ps}\right.$, see Table 1$)$ measured in DIMBQ, whereas the corresponding phosphorescence is apparently strong $\left(\Phi_{\mathrm{p}} \approx 0.3\right)$. After a long period of photolysis, when $>90 \%$ of the hydrogen-bonded enol species disappeared while monitoring the keto-tautomer fluorescence, very weak fluorescence at a peak maximum of $425 \mathrm{~nm}$ was indeed resolved for DIHBQ (inset B of Figure 6) under a high laser-excitation intensity (i.e., $\sim 35 \mathrm{~mJ} / \mathrm{cm}^{2}$ ). The lifetime was measured to be as short as $\sim 50$ ps (see Table 1 ). Similar heavyatom-enhanced decay dynamics of phosphorescence $\left(\tau_{\mathrm{p}} \approx 1.5\right.$ $\times 10^{-3} \mathrm{~s}$ ) were observed in the photolysis product (i.e., the 
TABLE 1: Photophysical Properties of HBQ and DIHBQ and Their Corresponding Methylated Derivatives in $298 \mathrm{~K}$ and $77 \mathrm{~K}$ Methylcyclohexane

\begin{tabular}{|c|c|c|c|c|c|c|c|}
\hline & \multirow{2}{*}{$\frac{\text { absorption }}{\lambda_{\max }(\mathrm{nm})^{a}}$} & \multicolumn{3}{|c|}{ fluorescence } & \multicolumn{3}{|c|}{ phosphorescence } \\
\hline & & $\lambda_{\max }(\mathrm{nm})$ & $\Phi_{\text {obs }}$ & $\tau_{\mathrm{f}}(\mathrm{ns})$ & $\lambda_{\max }(\mathrm{nm})$ & $\Phi_{\mathrm{obs}}{ }^{b}$ & $\tau_{\mathrm{p}}(\mathrm{s})^{b}$ \\
\hline \multirow[t]{3}{*}{ HBQ } & 380 & $403^{c}$ & & $3.00^{b}$ & $475^{c}$ & & 1.20 \\
\hline & & $625^{a}$ & $2.1 \times 10^{-3 a}$ & $0.30^{a}$ & & & \\
\hline & & $607^{b}$ & $6.3 \times 10^{-3 b}$ & $0.54^{b}$ & & & \\
\hline \multirow[t]{2}{*}{ MBQ } & 360 & $368^{a}$ & $0.05^{a}$ & $0.90^{a}$ & $466^{b}$ & & \\
\hline & & $367^{b}$ & & $3.33^{b}$ & & & \\
\hline \multirow[t]{3}{*}{ DIHBQ } & 395 & $425^{c}$ & & $0.05^{b}$ & $505^{c}$ & & $1.52 \times 10^{-3}$ \\
\hline & & $640^{a}$ & $1.8 \times 10^{-3 a}$ & $0.22^{a}$ & $735^{b}$ & $1.3 \times 10^{-5}$ & $1.75 \times 10^{-6}$ \\
\hline & & $610^{b}$ & $6.8 \times 10^{-3 b}$ & $0.60^{b}$ & & & \\
\hline DIMBQ & 375 & $400^{a}$ & $1.0 \times 10^{-4 a}$ & $0.12^{a}$ & $495^{b}$ & 0.3 & $1.20 \times 10^{-3}$ \\
\hline
\end{tabular}

${ }^{a} 298 \mathrm{~K} .{ }^{b} 77 \mathrm{~K} .{ }^{c}$ Photolysis products at $77 \mathrm{~K}$.

non-hydrogen-bonded enol form) of DIHBQ. In comparison, the phosphorescence lifetime of the non-hydrogen-bonded enol form was measured to be as long as $1.2 \mathrm{~s}$ in HBQ.

The photoinduced rupture of the intramolecular hydrogen bond may not be uncommon among ESIPT molecules. A similar mechanism has been proposed to explain the photolysis of salicylaldehyde (SA) in low-temperature solid matrixes. ${ }^{32}$ Nagaoka et al. ${ }^{33}$ have reported that except for the major channels of radiationless deactivation (i.e., proton-transfer reaction, intersystem crossing, and internal conversion) SA undergoes a minor photoisomerization channel, thus forming an open enolconformer. On the basis of FTIR analyses of vibrational modes for various deuterium isotope substitutions, Morgan et al. ${ }^{34}$ further resolved the open conformer to be a structure in which both the hydroxyl and aldehyde functional groups rotate by $180^{\circ}$.

When a CW Ar ${ }^{+}$laser $(362.5 \mathrm{~nm}$ ) was used as an excitation source, similar photolysis reactions were observed for both HBQ and DIHBQ in a $77 \mathrm{~K} \mathrm{MCH}$ glass. The results eliminate the photolysis mechanism that mainly results from the multiphoton event. In another approach, if the rupture of the intramolecular hydrogen bond occurred in the enol excited state, one would expect a similar reaction pattern in the keto ${ }^{1} \pi \pi^{*}$ state for which the lifetime $(\sim 600 \mathrm{ps})$ is much longer even than that $(\ll 15 \mathrm{ps})$ of the enol form. However, detailed excitation spectral analyses showed no existence of photolysis products with the absorption chromophore of $>420 \mathrm{~nm}$, indicating that photoisomerization did not take place in the keto form. Accordingly, the possibility that for HBQ and DIHBQ the electronically excited enol form undergoes an $\mathrm{O}-\mathrm{H}$ rotation in competition with the ultrafast ESIPT dynamics is discounted as well.

As shown in Scheme 1 (vide infra), both ESIPT and groundstate reverse proton-transfer reactions are highly exergonic. These results, in combination with the dominant $\mathrm{S}_{1}{ }^{\prime} \rightarrow \mathrm{S}_{0}{ }^{\prime}$ radiationless transition (see Table 1), led us to propose a more plausible mechanism that incorporates the formation of a vibrationally hot, hydrogen-bonded enol after a proton-transfer cycle. Subsequently, the highly exothermic energy dissipated from the radiationless transition generates the local heat, which thermally activates the $-\mathrm{OH}$ rotation in the glassy matrixes. The plausible product (i.e., a non-hydrogen-bonded enol form with the hydroxyl proton rotating out of the hydrogen-bonding configuration) is dynamically stable and is trapped in the glass matrix by the constraint of the solid environment. Upon fusion of the solvent, the product relaxes back to the thermodynamically more stable enol form possessing an intact hydrogenbonding configuration. Unlike SA, where both the $\mathrm{O}-\mathrm{H}$ and carbonyl functional groups can rotate simultaneously, ${ }^{34}$ pyridinic nitrogen in HBQ and DIHBQ is fixed in a planar geometry. Thus, the photolysis product (i.e., the enol conformer) seems to be incorporated only with the rotation of hydroxyl proton, though the actual degree of rotation is not accessible at this stage.

3.3. Energy Diagram. With the above experimental results, we herein attempt to construct an energy diagram regarding an overall proton-transfer cycle in DIHBQ. As shown in Scheme 1 , the energy of the $S_{0}$ state of the enol tautomer has been arbitrarily set to $0 \mathrm{kcal} / \mathrm{mol}$. In addition, because of more informative data and less thermal perturbation, the construction of relative energy levels is done under the environment of a 77 $\mathrm{K}$ MCH glass. Scheme 1 also shows population and quantum yields of various photophysical pathways. The relative energy levels for $\mathrm{S}_{1}{ }^{\prime}$ and $\mathrm{T}_{1}{ }^{\prime}$ were taken directly from the peak maxima of keto fluorescence $(610 \mathrm{~nm})$ and phosphorescence $(735 \mathrm{~nm})$, respectively. Fluorescence $(425 \mathrm{~nm})$ and phosphorescence (505 $\mathrm{nm}$ ) of the non-hydrogen-bonded enol form were applied for the $S_{1}$ and $T_{1}$ states. One must be cautious here to make sure that the hydrogen-bonded enol form normally exhibits a bathochromic spectral shift in the singlet manifold with respect to the non-hydrogen-bonded conformer. The relative energy gap between the enol $\left(\mathrm{S}_{0}\right)$ and keto $\left(\mathrm{S}_{0}{ }^{\prime}\right)$ forms in the ground state is experimentally unobtainable because of the ultrafast groundstate reverse proton transfer and thus has to be accessed through theoretical approaches. However, the large spin-orbit coupling factor limits ab initio calculations at higher-level basis sets. Alternatively, semiempirical approaches based on AM1 and PM3 methods were performed, resulting in $\mathrm{S}_{0}{ }^{\prime}$ being higher in energy than $\mathrm{S}_{0}$ by 8.7 and $11.8 \mathrm{kcal} / \mathrm{mol}$, respectively. By taking an average of $10.3 \mathrm{kcal} / \mathrm{mol}$ for the $\mathrm{S}_{0}-\mathrm{S}_{0}{ }^{\prime}$ energy gap, we depict the relative energy levels for the overall proton-transfer cycle in DIHBQ in Scheme 1. The $\mathrm{T}_{1}$ state of the enol form is estimated to be $\sim 7.42 \mathrm{kcal} / \mathrm{mol}$ higher in energy than the $\mathrm{T}_{1}{ }^{\prime}$ state of the keto tautomer, indicating that the $\mathrm{T}_{1}{ }^{\prime} \rightarrow \mathrm{T}_{1}$ reverse proton transfer is highly endergonic, which is consistent with the experimental results.

\section{Conclusions}

On the basis of the heavy-atom effect, the lowest-lying triplet state of the proton-transfer keto tautomer has been studied by its corresponding ultraweak phosphorescence at $735 \mathrm{~nm}$. The results, in combination with the photolysis experiment and theoretical approaches, lead to the establishment of relative energy levels during a proton-transfer cycle in different spin manifolds. The relaxation dynamics of $\mathrm{T}_{1}{ }^{\prime}$ were dominated by radiationless deactivation. The result is qualitatively consistent with the energy gap law, leading to the conclusion that the intramolecular radiationless deactivation constant should increase with the decreasing energy gap of the transition. ${ }^{25}$ The application of the iodine heavy-atom effect enhances both $\mathrm{S}_{1}{ }^{\prime} \rightarrow \mathrm{T}_{1}{ }^{\prime}$ intersystem crossing and $\mathrm{T}_{1}{ }^{\prime} \rightarrow \mathrm{S}_{0}{ }^{\prime}$ radiative decay rates, 
which, coupled with an ultrasensitive NIR detecting system, turns out to be crucial to the resolution of the spectroscopy and dynamics of the triplet states of ESIPT molecules.

Despite the strong intramolecular hydrogen bond formation in both HBQ and DIHBQ, O-H rotational enol conformers were produced during the photolysis. The similarity in the photolysis patterns between HBQ and SA derivatives leads us to propose the possibility of generalizing a mechanism incorporating photoinduced hydrogen bond breakage among ESIPT molecules in glassy matrixes, particularly for those possessing weak hydrogen bonds such as 3-hyroxyflavone. ${ }^{18 a}$ Our preliminary results have shown that in addition to the protic-solvent perturbation photoinduced breakage of the intramolecular hydrogen bond in 3-hydroxyflavones played a role in the prohibition of ESIPT reaction in $77 \mathrm{~K} \mathrm{MCH}$ glassy matrixes. ${ }^{35}$ Accordingly, one may have to exercise great caution when considering the application of ESIPT molecules to solid-state devices in which the operation is, in principle, based on the ESIPT mechanism.

Acknowledgment. This work was supported by the National Science Council NSC (90-2113-M-002-055). We thank the National Center for High-Performance Computing, Taiwan, for the use of their facility.

\section{References and Notes}

(1) For reviews on related subjects, see refs $2-11$

(2) Kasha, M. J. Chem. Soc., Faraday Trans. 2 1986, 82, 2379. 127.

(3) Kosower, E. M.; Huppert, D. Annu. Rev. Phys. Chem. 1986, 37,

(4) Barbara, P. F.; Walsh, P. K.; Brus, L. E. J. Phys. Chem. 1989, 93,

(5) Nagaoka, S.; Nagashima, U. Chem. Phys. 1989, 136, 153.

(6) Chou, P. T.; Martinez, M. L.; Studer, S. L. J. Phys. Chem. 1991, 95, 10306

(7) Formosinho, S. F.; Arnaut, L. G. J. Photochem. Photobiol., A 1993, 75,21 477.

(8) Douhal, A.; Lahmani, F.; Zewail, A. H. Chem. Phys. 1996, 207,

(9) Pfeiffer, M.; Lau, A.; Lenz, K.; Elsaesser, T. Chem. Phys. Lett. 1997, 268, 258

(10) Moriyama, M.; Kosuge, M.; Tobita, S.; Shizuka, H. Chem. Phys. 2000, 253, 91.

(11) Scheiner, S. J. Phys. Chem. A 2000, 104, 5898

(12) For example, see (a) Chou, P. T.; McMorrow, D.; Aartsma, T. J.; Kasha, M. J. Phys. Chem. 1984, 88, 4596. (b) Ernsting, N. P.; Nikolaus, B.
Appl. Phys. B 1986, 39, 155. (c) Acuña, A. V.; Amat-Guerri, F.; Catalán, J.; Costella, A.; Figuera, J.; Munoz, J. Chem. Phys. Lett. 1986, 132, 576. (d) Kasha, M. In Molecular Electronic Devices; Carter, F. L., Siatkowski, R. E., Wohltjen, H., Eds.; Elsevier Science Publishers: New York, 1988; pp 107-121. (e) Catalán, J.; del Valle, J. C. J. Am. Chem. Soc. 1993, 115, 4321. (f) Ferrer, M. L.; Acuña, A. U.; Amat-Guerri, F.; Costela, A.; Figuera, J. M.; Florido, F.; Sastre, R. Appl. Opt. 1994, 33, 2266. (g) Jones, G.; Rahman, M. A. J. Phys. Chem. 1994, 98, 13028. (h) Liphardt, M. Gooneskera, A.; Jones, B. E.; Ducharme, S.; Takacs, J. M.; Zhang, L. Science (Washington, D.C.) 1994, 263, 367. (i) Kuldová, K.; Corval, A.; Trommsdorff, H. P.; Lehn, J. M. J. Phys. Chem. A 1997, 101, 6850.

(13) Sytnik, A.; Gormin, D.; Kasha, M. Proc. Natl. Acad. Sci. U.S.A. 1994, 91, 8267, 11968.

(14) Sytnik, A.; del Valle, J. C. J. Phys. Chem. 1995, 99, 13028.

(15) Roshal, A. D.; Grigorovich, A. V.; Doroshenko, A. O.; Pivovarenko,

V. G.; Demchenko, A. P. J. Phys. Chem. A 1998, 102, 5907.

(16) Tarkka, R. M.; Zhang, X.; Jenekhe, S. A. J. Am. Chem. Soc. 1996, $118,9438$.

(17) Martinez, M. L.; Cooper, W. C.; Chou, P. T. Chem. Phys. Lett. 1992, 193, 151 .

(18) For example, see (a) Kasha, M.; McMorrow, D. J. Phys. Chem. 1984, 88, 2235. (b) Grellman, K. H.; Mordzinski, A.; Heinrich, A. Chem. Phys. 1989, 136, 201. (c) Schwartz, B. J.; Peteanu, L. A.; Harris, C. B. J. Phys. Chem. 1992, 96, 3591. (d) Tobita, S.; Yamamoto, M.; Kurahayashi, N.; Tsukagoshi, R.; Nakamura, Y.; Shizuka, H. J. Phys. Chem. A. 1998, 102, 5206.

(19) Chou, P. T.; Wei, C. Y. J. Phys. Chem. 1996, 100, 17059

(20) Chou, P. T.; Martinez, M. L. Radiat. Phys. Chem. 1993, 41, 373.

(21) Roberts, E. L.; Chou, P. T.; Alexander, T. A.; Agbaria, R. A.; Warner, I. M. J. Phys. Chem. 1995, 99, 5431.

(22) del Valle, J. C.; Catalán, J. Chem. Phys. 2001, 270, 1.

(23) Chou, P. T.; Chen, Y. C.; Yu, W. S.; Chou, Y. H.; Wei, C. Y.; Cheng, Y. M. J. Phys. Chem. A 2001, 105, 1731.

(24) McGlynn, S. P.; Azumi, T.; Kinoshita, M. Molecular Spectroscopy of the Triplet State; Prentice Hall: Englewood Cliffs, NJ, 1969; p 18.

(25) Sieband, W. J. Chem. Phys. 1967, 47, 2411.

(26) Klöpffer, W. Adv. Photochem. 1977, 10, 311.

(27) For recent studies of heavy atom effects on the ESIPT reaction, see Catalán, J.; Diaz, C. J. Phys. Chem. A 1998, 102, 323.

(28) Battino, R.; Rettich, T. R.; Tominaga, T. J. Phys. Chem. Ref. Data. 1983, 12, 163.

(29) Birks, J. B. Photophysics of Aromatic Molecules; Wiley: New York, $1970 ;$ p 313.

(30) (a) Schmidt, R.; Bodesheim, M. Chem. Phys. Lett. 1993, 213, 111. (b) Schmidt, R.; Bodesheim, M. J. Phys. Chem. 1994, 98, 2874. (c) Schmidt, R.; Bodesheim, M. J. Phys. Chem. 1995, 99, 15919.

(31) Schmidt, R.; Tanielian, C.; Dunsbach, R.; Wolff, C. J. Photochem. Photobiol., A 1994, 79, 11.

(32) Lamola, A. A.; Sharp, L. J. J. Phys. Chem. 1966, 70, 2634.

(33) Nagaoka, S.; Hirota, N.; Sumitani, M.; Yoshihara, K. J. Am. Chem. Soc. $1983,105,4220$.

(34) Morgan, M. A.; Orton, E.; Pimental, G. C. J. Phys. Chem. 1990, 94, 7927.

(35) Chou, P. T.; Wei, C. Y. Unpublished work. 